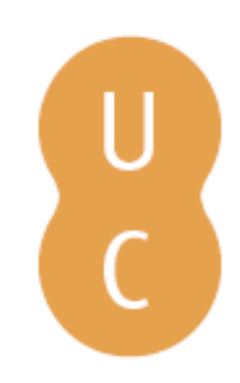

pnmbalina

\title{
Condicionantes da saúde mental e os instrumentos de avaliação de impactos
}

$\begin{array}{ll}\text { Autor(es): } \quad \text { Loureiro, Adriana; Lima, Joana; Partidário, Maria do Rosário; Santana, } & \text { Paula }\end{array}$

Publicado por: Imprensa da Universidade de Coimbra

URL

persistente: URI:http://hdl.handle.net/10316.2/38275

DOI: $\quad$ DOI:http://dx.doi.org/10.14195/978-989-26-1105-1_1

Accessed : $\quad$ 26-Apr-2023 13:10:09

A navegação consulta e descarregamento dos títulos inseridos nas Bibliotecas Digitais UC Digitalis, UC Pombalina e UC Impactum, pressupõem a aceitação plena e sem reservas dos Termos e Condições de Uso destas Bibliotecas Digitais, disponíveis em https://digitalis.uc.pt/pt-pt/termos.

Conforme exposto nos referidos Termos e Condições de Uso, o descarregamento de títulos de acesso restrito requer uma licença válida de autorização devendo o utilizador aceder ao(s) documento(s) a partir de um endereço de IP da instituição detentora da supramencionada licença.

Ao utilizador é apenas permitido o descarregamento para uso pessoal, pelo que o emprego do(s) título(s) descarregado(s) para outro fim, designadamente comercial, carece de autorização do respetivo autor ou editor da obra.

Na medida em que todas as obras da UC Digitalis se encontram protegidas pelo Código do Direito de Autor e Direitos Conexos e demais legislação aplicável, toda a cópia, parcial ou total, deste documento, nos casos em que é legalmente admitida, deverá conter ou fazer-se acompanhar por este aviso.

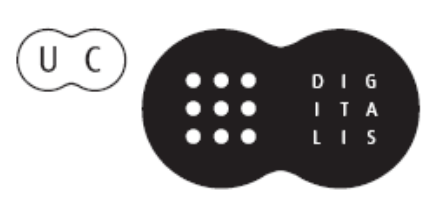




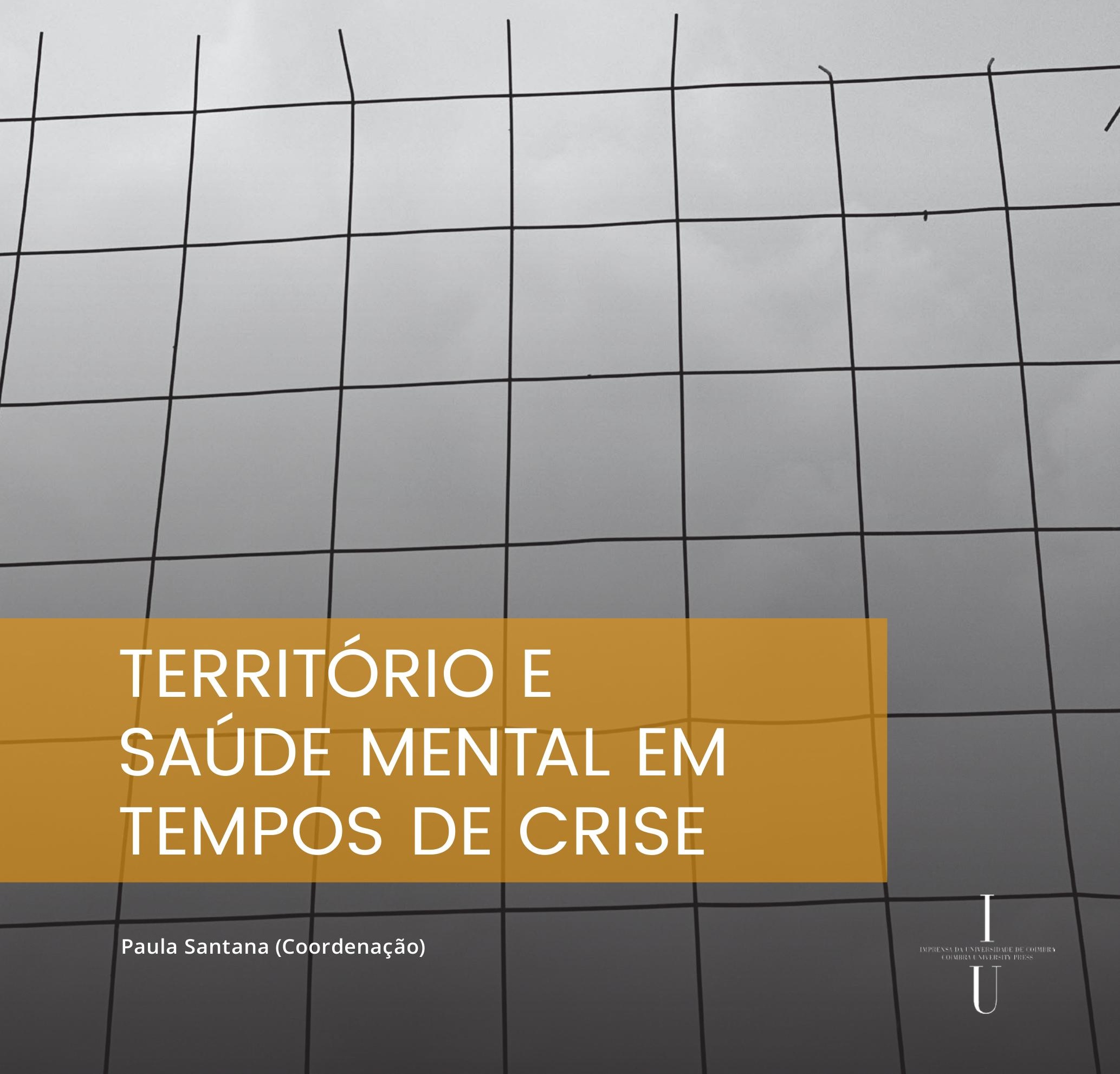




\subsection{Condicionantes da saúde mental e os instrumentos de avaliação de impactos}

Adriana Loureiro ${ }^{(1,2)}$, Joana Lima ${ }^{(3)}$, Maria do Rosário Partidário ${ }^{(4)}$ e Paula Santana ${ }^{(1,2)}$

(1) Centro de Estudos de Geografia e Ordenamento do Território (CEGOT), Universidade de Coimbra; (2) Departamento de Geografia e Turismo, Universidade de Coimbra; (3) Departamento de Sociologia, Universidade de Oxford; (4) Instituto Superior Técnico,

Universidade de Lisboa.

DOI: http://dx.doi.org/10.14195/978-989-26-1105-1_1

A saúde mental é uma dimensão intrínseca e menos valorizada da saúde e do bem-estar do indivíduo. Para além de fatores biológicos, genéticos e de circunstâncias pessoais, o(s) ambiente(s) (lugar(es) onde o indivíduo nasce, se desenvolve e envelhece) parece $(\mathrm{m})$ condicionar a saúde e bem-estar mental. Este capítulo explora a evidência científica que procura demonstrar como o lugar influencia o estado de saúde mental dos indivíduos e das comunidades, a diversas escalas e para diferentes populações. A literatura sugere que a distribuição destes fatores que condicionam a saúde mental pode explicar desigualdades e iniquidades em saúde bem como em desenvolvimento e bem-estar. Esta abordagem é particularmente relevante em períodos de crise económica, existindo uma tendência para muitos destes fatores serem significativamente afetados, acentuando-se, em consequência, as desigualdades geográficas e sociais na saúde mental.

Neste texto são ainda apresentados instrumentos disponíveis para avaliar os impactos na saúde mental que têm por base os seus condicionantes.
Mental health is an inherent, and underrated, component of people's overall health and wellbeing. In addition to biologic and genetic factors and personal circumstances, the built and natural environments where an individual is born, lives and ages may crucially determines their health and wellbeing.

This chapter explores the scientific evidence that aims to demonstrate how and to what extent the physical and social environment influence the mental health of individuals and communities, on different scales and on different populations. The literature suggests that the distribution of these determinants of mental health can explain inequalities and inequities in health and wellbeing more broadly. This approach is particularly relevant in periods of economic crisis when many of these determinants are severely affected. Consequently, social and geographical inequalities in mental health are polarized.

Finally, the chapter offers a discussion of the instruments at our disposal to evaluate the mental health impacts of changes in its determinants. 


\section{Introdução}

A saúde mental é intrínseca à saúde e ao bem-estar do indivíduo (Patel et al., 2010; Prince et al., 2007). O entendimento da saúde mental não se esgota apenas na ausência de doença mental. Segundo a Organização Mundial de Saúde (2014), é um estado de bem-estar em que cada indivíduo percebe o seu próprio potencial, conseguindo lidar com as tensões normais da vida, trabalhar de forma produtiva e frutífera e com capacidade de contribuir para a sua comunidade. Nesta definição estão incluídos conceitos de bem-estar subjetivo, autoeficácia percecionada, autodeterminação, autonomia, competência social, dependência intergeracional e autorrealização do potencial intelectual e emocional (WHO, 2001). Não existe apenas uma definição de saúde mental. Esta depende em muito da influência cultural na construção do conceito, da subjetividade do próprio conceito e das diversas perspetivas profissionais.

A saúde mental do indivíduo está dependente das suas vivências, estando, em parte, relacionada com os seus lugares e ambientes de vida (residência, estudo, trabalho, lazer, recreação, mobilidade). Para além de fatores biológicos, genéticos e de circunstâncias pessoais, o ambiente no território onde o indivíduo nasce, se desenvolve e envelhece é determinante para a sua saúde e bem-estar mental (WHO \& Calouste Gulbenkian Foundation, 2014). O ambiente é assim entendido num âmbito mais alargado e holístico, interrelacionando-se com o território e suas características sociais, económicas, culturais, físicas, institucionais, entre outras.

A evidência científica tem demonstrado que o território influência o estado de saúde mental dos indivíduos e das próprias comunidades, a diversas escalas e para diferentes populações, ao considerar a inter-relação complexa de múltiplos fatores que determinam o valor particular de cada lugar (Barcelos \& Bastos, 1996; Santana, 2005). Esta abordagem, baseada na distribuição dos fatores determinantes da saúde mental, tem sido utilizada para explicar desigualdades e iniquidades em saúde (Nogueira,
2006; Santana, Santos \& Nogueira, 2009). As crises económicas constituem uma alteração da interação normal entre os fatores determinantes da saúde mental, produzindo frequentemente um agravamento das condições sociais e económicas (Stuckler \& Basu, 2013).

Neste âmbito, são colocados desafios à governança dos territórios, nomeadamente a nível local, na definição, implementação, avaliação e monitorização de estratégias e políticas com impactos na saúde e bem-estar mental das populações e na equidade em saúde (Fischer, Matuzzi \& Nowacki, 2010; De Snyder et al., 2011; Harris-roxas et al., 2012; Todman et al., 2012). As ações de intervenção no território, ordenamento, planeamento e design, com atuação em e.g. recursos, infraestruturas e condições sociais, geram impactos na saúde de uma comunidade, aumentando ou diminuindo as desigualdades em saúde (Santana, 2005).

Nos últimos anos têm sido desenvolvidas abordagens estratégicas que permitem promover os processos de desenvolvimento dos territórios com base nas suas características (Partidário, 2012). Existem instrumentos com capacidade de gerar conhecimento sistemático sobre os fatores que condicionam ou promovem a saúde, que podem apoiar os agentes locais na sua atuação politico-estratégica com efeitos na mitigação da doença mental e na promoção de bem-estar (Nowacki, Martuzzi \& Fischer, 2009; Fischer, Matuzzi \& Nowacki, 2010; Birley, 2011; Harris-roxas et al., 2012; Partidário, 2012).

Este capítulo pretende apresentar e enquadrar, com base em evidência científica presente na literatura, os condicionantes da saúde mental (características dos territórios e dos ambientes de vida dos indivíduos), bem como os fatores que derivam de contextos de crise económica. Refere-se também a instrumentos de avaliação de impactos na saúde mental - Avaliação de Impactos na Saúde e Avaliação Ambiental Estratégica, apresentando os seus conceitos, objetivos e princípios orientadores. 


\section{Condicionantes}

\section{da saúde mental'}

Evidência empírica, com destaque para trabalhos de investigação que têm utilizado análises estatísticas multifatoriais, referem que independentemente das características individuais, o contexto (território e ambientes de vida do indivíduo) podem influenciar a sua saúde e bem-estar mental (Drukker \& van Os, 2003; Kubzansky et al., 2005; Skapinakis et al., 2005; Araya et al., 2006; Fone \& Dunstan, 2006; Sundquist \& Ahlen, 2006; Dupéré \& Perkins, 2007; Fone et al., 2007a; Fone et al., 2007b; Lee, 2009; Hamano et al., 2010; Rios, Aiken \& Zautra, 2012). Considerando a amplitude e a abrangência dos conceitos em causa, a produção de conhecimento científico neste âmbito tem ocorrido através da investigação de diversos resultados em saúde mental: sofrimento psicológico, depressão/sintomas depressivos, ansiedade/sintomas de ansiedade, sintomas psicossomáticos, prevalência de perturbações mentais, internamentos hospitalares psiquiátricos, óbitos por suicídio e lesões autoinfligidas, qualidade de vida/satisfação com a vida, bem-estar e felicidade.

As características dos territórios (onde se nasce, cresce, vive, trabalha e envelhece), funcionando como "determinantes contextuais", podem produzir impactos positivos ou negativos na saúde em geral (Macintyre \& Ellaway, 2000) e na saúde mental individual e coletiva (WHO, 2008, 2013; WHO \& Calouste Gulbenkian Foundation, 2014). dos determinantes da saúde mental, afirmam que para se atingir uma melhor saúde mental terão de ser introduzidas melhorias nas condições de vida, nomeadamente nas características do ambiente físico e construído, socioeconómico e de interação social e cultural em que os indivíduos se inserem.

Estas condições podem representar riscos mais elevados de doença mental em alguns grupos sociais, considerando a sua maior exposição e vulnerabilidade a ambientes sociais, económicos, físicos e construídos desfavoráveis (WHO \& Calouste Gulbenkian Foundation, 2014).

O quadro seguinte sintetiza as características do contexto que influenciam a saúde mental referidas na literatura. De facto, as desigualdades na vivência

Quadro 1. Condicionantes contextuais (ambiente físico e construído, ambiente socioeconómico e de interação social e cultural) da saúde mental

\begin{tabular}{|c|c|c|}
\hline $\begin{array}{l}\text { Características } \\
\text { do ambiente físico } \\
\text { e construído }\end{array}$ & $\begin{array}{l}\text { Características } \\
\text { do ambiente } \\
\text { socioeconómico }\end{array}$ & $\begin{array}{l}\text { Características do } \\
\text { ambiente de interação } \\
\text { social e cultural }\end{array}$ \\
\hline Habitação & Pobreza & $\begin{array}{l}\text { Redes sociais e } \\
\text { de apoio social }\end{array}$ \\
\hline Saneamento & Rendimento & Capital social \\
\hline Poluição & Emprego/Desemprego & Coesão social \\
\hline Mobilidade e transportes & Educação & Identidade e pertença \\
\hline Espaço verde e azul & Segurança alimentar & $\begin{array}{c}\text { Racismo e } \\
\text { discriminação social }\end{array}$ \\
\hline $\begin{array}{c}\text { Dispersão/concentração } \\
\text { da população }\end{array}$ & $\begin{array}{c}\text { Estatuto } \\
\text { socioeconómico }\end{array}$ & Justiça \\
\hline $\begin{array}{c}\text { Desenho do } \\
\text { espaço urbano }\end{array}$ & & \\
\hline $\begin{array}{l}\text { Manutenção do } \\
\text { espaço urbano }\end{array}$ & & \\
\hline Uso do solo & & \\
\hline $\begin{array}{c}\text { Acesso a } \\
\text { equipamentos e serviços }\end{array}$ & & \\
\hline Segurança & & \\
\hline Presença de pichagem & & \\
\hline
\end{tabular}

Fonte: elaborado a partir da literatura revista

Alguns autores (Evans, 2003; Almedom, 2005; Sundquist \& Ahlen, 2006; Fone \& Dunstan, 2006; Miles, Coutts \& Mohamadi, 2012; Todman et al., 2013), apoiando-se nesta abordagem holística dos ambientes e das suas características (quadro 1) podem traduzir diferenças na saúde mental dos indivíduos e das comunidades, que potenciam iniquidades pelo seu carácter injusto e evitável (WHO, 2008).

1. Esta secção foi adaptada da publicação: Loureiro, A.; Costa, C. \& Santana, P. (2015), "Determinantes Contextuais da Saúde Mental", in Dimenstein, M. (org.), Condições de Vida e Saúde Mental em Assentamentos Rurais, Universidade Federal do Rio Grande do Norte, Editora Intermeios, Natal (no prelo). 
Todavia, há três aspetos a ter em conta nesta abordagem. Por um lado, a relação de influência raramente é direta e a maioria dos impactos do lugar na saúde mental expressam-se por relações indiretas (Burton, 2015). Por outro lado, a ação num determinante ambiental poderá ter impactos noutros determinantes, motivando alterações em cadeia, cumulativas, através de ações de sobreposição e interação (Nogueira, Santana \& Santos, 2007). Por fim, a interação indivíduo-território/ ambiente-indivíduo pode não ocorrer do mesmo modo, mesmo considerando indivíduos com as mesmas características biológicas e genéticas.

\subsection{Ambiente físico e construído}

O Lugar é uma dimensão importante para a saúde mental dos indivíduos e das comunidades (Curtis, 2010). O ambiente físico e construído que nos envolve pode afetar a nossa saúde e bem-estar mental, tendo, por vezes, a capacidade de nos "animar" ou "levantar o espírito" ou, por outro lado, de nos deixar "deprimidos" ou "claustrofóbicos" (Burton, 2015). Neste sentido, o ambiente físico e construído pode atuar sobre os comportamentos e perceções do indivíduo influenciando, por exemplo, a prática de atividade física, a interação social, o contacto com a natureza, a acessibilidade e mobilidade, o sentimento de pertença e a segurança (Brown, Learmonth \& Mackereth, 2015; Burton, 2015).

Na última década, vários estudos científicos têm evidenciado associações entre resultados em saúde mental e características ou elementos do ambiente físico e construído como os relativos à habitação (e.g. forma e design, isolamento térmico e acústico, lotação, luminosidade) (Brown, 1995; Weich et al., 2002; Drukker \& van Os, 2003; Evans, Wells \& Moch, 2003; Galea et al., 2005; Vlahov et al., 2005; Brown et al., 2009; Bond et al., 2012; Barahmand, Shahbazi \& Shahbazi, 2013), à densidade residencial e populacional (Walters et al., 2004; Peen et al., 2007), à qualidade ambiental (e.g. recolha e tratamento de resíduos, ruído, qualidade do ar e da água) (Araya et al., 2007; Thomas et al., 2007), ao acesso a infraestruturas e serviços (e.g. espaços verdes, cuidados de saúde) (Takano, 2002 ; Guite, Clark \& Ackrill, 2006;
Araya et al., 2007; Gary, Stark \& LaVeist, 2007; Sheppard et al., 2012), ao desenho do espaço urbano e usos do solo (e.g. espaço público, configuração das ruas, uso misto, pedonalidade, presença do espaço verde) (Araya et al., 2007; Berke et al., 2007; Annerstedt et al., 2012; Bond et al., 2012; Sheppard et al., 2012), à segurança (e.g. crime) (Whitley \& Prince, 2005; Araya et al., 2007; Cromley, Wilson-Genderson \& Pruchno, 2012; Sheppard et al., 2012; Wilson-Genderson \& Pruchno, 2013) e à manutenção do espaço público (e.g. presença de grafíti não autorizados, sinais de vandalismo) (Semenza \& Krishnasamy, 2007).

Em síntese, a literatura tem sugerido que piores resultados em saúde mental estão relacionados com: habitação sobrelotada, com pouca entrada de luz natural, fraco isolamento térmico e acústico, inestética do espaço construído (Drukker \& van Os, 2003; Bond et al., 2012; Barahmand, Shahbazi \& Shahbazi, 2013); elevadas taxas de crime, perceção de insegurança e crime (Whitley \& Prince, 2005; Gary, Stark \& LaVeist, 2007; Cromley, Wilson-Genderson \& Pruchno, 2012; Sheppard et al., 2012; Wilson-Genderson \& Pruchno, 2013); inexistência visual de espaço verde ou de espaço azul (Sugiyama et al., 2008; Annerstedt et al., 2012); acesso inadequado a infraestruturas e serviços (Guite, Clark \& Ackrill, 2006; Gary, Stark \& LaVeist, 2007); densidade residencial e populacional muito elevadas (Walters et al., 2004; Peen et al., 2007) ou muito baixas (Hempstead, 2006; Jagodic, Agius \& Pregelj, 2012; Santana et al., 2015); elevados níveis de ruído (Guite, Clark \& Ackrill, 2006) e baixa pedonalidade da área de residência/trabalho (Berke et al., 2007).

\subsection{Ambiente socioeconómico}

A relação entre as características socioeconómicas do contexto de vida do indivíduo e os seus resultados em saúde mental tem suscitado inúmeras investigações.

Áreas pobres, de baixo estatuto e de privação socioeconómica são espaços onde são geradas poucas oportunidades, onde as situações de vulnerabilidade se multiplicam, conjugando-se 
simultaneamente vários fatores (Kaplan, 1996; Nogueira, 2007) que potenciam o risco de piores resultados em saúde mental (Stafford, 2003; Murali, 2004). Genericamente classificam-se como lugares em declínio económico, social e demográfico, tendo por base processos de desqualificação que assentam em mecanismos de acumulação e retroação, ocupados por populações carenciadas e empobrecidas, degradados habitacionalmente, desprovidos de equipamentos públicos e/ou privados, onde se incluem os do comércio e serviços e, consequentemente, tendem a constituir-se como territórios de baixa interação social e fraca utilização do espaço público (Nogueira, 2007).

Existe evidência científica que identifica associações estatísticas entre piores resultados em saúde e bem-estar mental e as características sociais e económicas das áreas de residência, destacando: baixos rendimentos (Zhang, Ho \& Woo, 2005; Sundquist \& Ahlen, 2006; Fone et al., 2007b; Orpana, Lemyre \& Gravel, 2009;Roberts, Abbott \& Mckee, 2010; Fukuda \& Hiyoshi, 2012), níveis elevados de desemprego ou muito baixa atividade económica (Breslin \& Mustard, 2003; Comino et al., 2003; Artazcoz et al., 2004; Fone \& Dunstan, 2006; Fone et al., 2007a; Thomas, Benzeval \& Stansfeld, 2007; Roberts, Abbott \& Mckee, 2010; Fukuda \& Hiyoshi, 2012; Córdoba-Doña et al., 2014) e falta de capacidade económica para aceder a uma alimentação saudável (Cannuscio, Weiss \& Asch, 2010; Yang \& Matthews, 2010).

Considerando a multidimensionalidade destes conceitos, alguns estudos utilizam medidas compósitas de estatuto socioeconómico (Pringle et al., 2000). Estas medidas agregam um conjunto muito diverso de variáveis relativas ao Lugar: emprego/desemprego, escolaridade, ocupação profissional, estado civil, tipologia da família, participação cívica, acessibilidade geográfica a serviços, acesso a meios de transporte e características da habitação e da alimentação. Neste sentido, pior saúde mental está associada a ambientes com baixo estatuto socioeconómico (Huurre et al., 2005; Lorant et al., 2007; Murata et al., 2008; Myer et al., 2008; Barahmand, Shah- bazi \& Shahbazi, 2013), de pobreza (Ostir et al., 2003; Kubzansky et al., 2005; Ludwig et al., 2012; Cromley, Wilson-Genderson \& Pruchno, 2012) e de privação socioeconómica (Drukker \& van Os, 2003; Walters et al., 2004; Skapinakis et al., 2005; Fone \& Dunstan, 2006; Santana et al., 2015).

\subsubsection{Ambiente socioeconómico em períodos de crise económica: evidência e mecanismos para a saúde mental}

\section{Evidência}

A crise económica mundial que se instalou na sequência da crise financeira de 2008, trouxe consigo o deteriorar das características do ambiente socioeconómico para uma parte significativa da população, não só nos Estados Unidos, onde a crise teve início, mas também na Europa. Como tal, o estudo dos efeitos dos ciclos económicos na saúde têm-se intensificado nos últimos anos, significando um maior leque de consequências documentadas para a saúde mental.

Recessões e outras crises económicas em décadas anteriores estiveram associadas com, por exemplo, uma diminuição da mortalidade geral, e com uma particular diminuição da mortalidade associada a acidentes de tráfico (Ruhm 2000, 2003, 2005). A exceção nessa tendência decrescente durante períodos de contração económica é a mortalidade por suicídio que tende a aumentar nestas circunstâncias (Ruhm, 2000, 2003, 2005). O aumento nas taxas de suicídio, já verificado em períodos de crise anteriores, parece ser de novo uma característica da atual crise que atravessamos. Por exemplo, Stuckler e outros (2009) calculam que na Europa um aumento de $1 \%$ na taxa de desemprego está associado com um aumento de $0,79 \%$ na taxa de suicídio em faixas etárias inferiores a 65 anos de idade. Adicionalmente, estes autores calculam que um aumento do desemprego de 3\% tem o maior efeito no aumento de suicídios (4,45\%, IC95\% $0,65-8,24)$ e no aumento de mortes resultantes de abuso de álcool em indivíduos de idade laboral (Stuckler et al., 2009). 
Outros indicadores de saúde mental também tendem a variar em resposta a oscilações na economia verificando-se um claro aumento em diversos diagnósticos, nomeadamente de depressão e ansiedade. O consumo de álcool, frequentemente associado a problemas de saúde mental, também tem sido objeto de estudo durante crises económicas. Intuitivamente teria sentido assumir que pudesse existir uma redução no consumo de álcool e de outros produtos nocivos, como tabaco, devido à redução do poder de compra verificado durante crises económicas. No entanto, um escrutínio mais detalhado do efeito da atual crise económica nos Estados Unidos, por exemplo, revela que, enquanto ao nível do agregado se verifica, de facto, uma redução do consumo de bebidas alcoólicas, em sectores da população mais vulneráveis e com uma rede de apoio mais fraca (como homens solteiros de idade inferior a 30 anos que perderam o emprego), este consumo aumenta desproporcionalmente (Bor et al., 2013). Este facto conduz a um aumento de internamentos devido a consumo excessivo de álcool (Bor et al., 2013). Outros autores descrevem um padrão similar no Reino Unido onde, apesar da tendência geral de diminuição do consumo de álcool durante a crise, se verifica um aumento significativo de consumo extremo entre homens desempregados (Harhay et al., 2014).

\section{Mecanismos}

O efeito das crises económicas na saúde mental atua fundamentalmente através de dois mecanismos que podem coexistir. O primeiro entra em ação através de choques económicos. Estes choques advêm de uma série de fatores que vão desde a perda de emprego, perda de poupanças, perda da casa ou expulsão da mesma, dívidas ou insolvência. O caso espanhol proporciona uma boa ilustração deste mecanismo. Um estudo (Gili et al., 2012) que acompanhou uma coorte de indivíduos na rede de cuidados de saúde primários no período pré e pós-crise verificou um aumento nos diagnósticos de depressão, ansiedade, transtornos somatoformes, transtornos da alimentação e abuso de álcool. Mas não só. Os autores descreveram adicionalmente um aumento do risco relativo de depressão entre os indivíduos que se encontravam em situação de despejo eminente, devido ao não pagamento de hipotecas, bem como em situação de desemprego. Podemos encontrar um outro exemplo deste mecanismo na Grécia, onde Barlow e outros (2015) documentaram, também usando uma coorte de indivíduos seguidos no período pré e pós-crise, um aumento do risco relativo (risco relativo de 1,61 ) de declínio da saúde auto reportada associado com a perda de emprego (Barlow et al., 2015).

A segunda via atua através de medidas de austeridade fiscal que reduzem os recursos disponíveis para manter sistemas de saúde e sistemas de segurança social. Os sistemas que, em circunstâncias normais, garantiriam uma rede de apoio ao individuo com problemas de saúde mental e impediriam a degradação da saúde e a possibilidade de uma vida normal, estão debilitados e incapazes de proporcionar esse nível de proteção. Um bom exemplo desta via são os programas de reinserção laboral ativa que ajudam os desempregados a melhorar a sua empregabilidade, dando-lhes não só formação mas também acesso a novas oportunidades de emprego. Estes programas têm efeitos comprovados na proteção da saúde mental verificados em intervenções nos Estados Unidos (van Ryn \& Vinokur, 1992; Vinokur et al., 2000) e na Finlândia (Vuori et al., 2002). Quanto à União Europeia, um estudo de 2009 (Stuckler et al., 2009) mostra que por cada aumento de 10 USD em proteção social investido em programas ativos de reinserção, o efeito do aumento do desemprego na taxa de suicídios é atenuado em 0,038\% (IC95\%, 0,004 - 0,071).

Apesar da proliferação de estudos que ilustram os vários mecanismos, não há consenso na literatura científica sobre a direção causal da associação. A grande maioria destes estudos usa o desemprego como indicador da gravidade da crise, o que causa alguns problemas já que este pode confundir a relação causa-efeito. Há duas interpretações preponderantes. Por um lado, temos a escola que defende que o desemprego causa a perda de saúde mental. Por exemplo, uma pessoa que perde o seu emprego perde não só a sua fonte de rendimento e a segurança material que este proporciona mas também um sentimen- 
to de pertença e de utilidade para a sociedade. Adicionalmente, o horário de trabalho também proporciona uma estrutura clara para o dia-adia. Consequentemente, a perda de emprego traz consigo o empobrecimento material e emocional. Além disso, a rede social associada ao posto de trabalho pode ser enfraquecida por uma falta de contacto diário, o que contribui para o quadro de isolamento. Igualmente, um aumento nas cifras de trabalho precário em pessoas empregadas que ainda assim estão abaixo do limiar da pobreza pode levar a uma preocupação constante com questões financeiras. Este desgaste leva a um elevado e constante nível de pressão e stress que invariavelmente culmina num desgaste da saúde mental. Há estudos que suportam esta teoria. A nível técnico, a evidência mais sólida a favor é aquela que se apoia em estudos longitudinais em que o individuo é seguido ao longo dos anos durante períodos de emprego e períodos subsequentes de desemprego. Murphy e Athanasou (1999) levaram a cabo uma meta-análise da literatura a fim de responder à pergunta de relação causal. Estes autores reviram a literatura que respondia à questão do efeito de entrar no mercado de trabalho, e a que respondia a questão do efeito de perder o emprego, na saúde mental e concluem que entrar no mercado de trabalho, após um período de desemprego, tem um efeito protetor na saúde mental. Paralelamente, a perda de emprego precede o despoletar de problemas de saúde mental (Murphy \& Athanasou, 1999).

A outra escola defende que a direção causal tem o sentido inverso: num clima de insegurança laboral criado pela crise económica, em que tanto o sector público como o privado aumentam as taxas de despedimentos ou reduzem o número de renovações de contratos, os indivíduos com problemas de saúde mental serão serão os primeiros a ser despedidos. Isto pode ser explicado pelo facto de estes indivíduos, no passado, apresentarem menor produtividade ou maior frequência de absenteísmo laboral em virtude de sofrerem de ansiedade, depressão ou qualquer outro diagnóstico de saúde mental. Outros autores propõem outro mecanismo: pessoas com problemas de saúde mental podem apresentar um maior consumo de álcool bem como revelar maior proba- bilidade de ter um comportamento agressivo, o que pode prejudicar a harmonia no local de trabalho e consequentemente aumentar o risco de desemprego (Catalano \& Bellows, 2005).

\subsection{Ambiente de interação social e cultural}

A forma de funcionamento da comunidade e a sua organização social são fatores que produzem efeitos na saúde mental da população. A influência positiva destas dimensões pressupõe a interação entre indivíduos, ou seja, a existência de relações de confiança, ajuda e cooperação, o compromisso cívico, a partilha de uma identidade comum, a presença de um sentimento de pertença a uma sociedade/comunidade e a adesão/união dos cidadãos (Baum \& Ziersch, 2003). Barton, Grant \& Guise (2003) afirmam que a existência de redes locais de suporte mútuo e confiança são fulcrais para a saúde e bem-estar de uma comunidade.

Alguns autores evidenciam a associação entre os resultados em saúde mental e: i) variáveis relacionadas com a participação política e confiança nas instituições, revelada pela população (Poblete, Sapag \& Bossert, 2008; Hamano et al., 2010), ii) as relações entre vizinhos (Fone et al., 2007b; Myer et al., 2008; Poblete, Sapag \& Bossert, 2008; Rios, Aiken \& Zautra, 2012), iii) o associativismo e participação em organizações (Dupéré \& Perkins, 2007; Gary, Stark \& LaVeist, 2007; Hamano et al., 2010; Myer et al., 2008), iv) a participação em grupos religiosos (Corrêa et al., 2010), v) o acesso a apoio/suporte social (Lee, 2009; Corrêa et al., 2010; Roberts, Abbott \& Mckee, 2010; Chou, 2012; Tsai \& Thompson, 2013), vi) o voluntariado (Poblete, Sapag \& Bossert, 2008) e vii) o isolamento social (Quinn \& Biggs, 2010). Neste sentido, a literatura científica tem revelado que piores resultados em saúde mental estão associados a ambientes com redes sociais fracas ou inexistentes (Quinn \& Biggs, 2010; Roberts, Abbott \& Mckee, 2010; Rios, Aiken \& Zautra, 2012), baixo capital social (Myer et al., 2008), baixa coesão social (Fone et al., 2007b). 


\section{Avaliação estratégica de impactos na saúde mental}

Ordenar e planear lugares mais saudáveis, capazes de promover a saúde e o bem-estar dos seus habitantes é hoje um dos grandes desafios colocados aos atores locais nos seus vários domínios de intervenção no território. Considerando a abordagem complexa, subjetiva, multifatorial e holística que é implícita à saúde e ao território, o planeamento territorial deverá basear-se no conhecimento das características ambientais com efeitos potenciais na saúde, no bem-estar humano e na relação que estabelecem entre eles (Santana, 2009), constituindo-se assim como fatores ambientais da saúde. Neste sentido, os processos de planeamento urbano saudável terão de ter em conta a utilização de variáveis, metodologias e instrumentos adequados para a operacionalização do conceito de território saudável.

Atualmente, a inclusão deste quadro conceptual e relacional nos processos locais de planeamento e ordenamento é obrigatório (Nowacki et al. 2009 e Circular informativa $n^{\circ}$ 36/DA de 9 de outubro de 2009), exigindo-se, no quadro das avaliações de impacto ambiental e de Avaliação Ambiental Estratégica que a temática "saúde" passe a ir mais além do que os aspetos tradicionais relacionados com rede de equipamentos de cuidados em saúde (Santana, Costa \& Loureiro, 2014) e seja abordada de forma mais integrada intersetorialmente (Partidário \& Jesus, 2007). Partidário e Jesus (2003) referem as vantagens na sua adoção como: i) a inclusão da saúde na previsão de impactos e prevenção de problemas, ii) a saúde ser considerada um elemento necessário para a sustentabilidade, iii) a saúde ser uma preocupação fundamental da população envolvida, e iv) os ganhos em saúde constituírem uma vantagem política.

Hoje existem abordagens e instrumentos metodológicos integradores e orientados para a sustentabilidade que pretendem avaliar impactos das opções de desenvolvimento tendo em conta as características do território, apoiando os pro- cessos de decisão - Avaliação de Impactos na Saúde e Avaliação Ambiental Estratégica (Harris-Roxas et al., 2012; Partidário, 2012).

A Avaliação de Impactos na Saúde é definida pela Organização Mundial de Saúde como uma combinação de procedimentos, métodos e instrumentos que sistematicamente julgam os potenciais efeitos, muitas vezes não intencionais, de políticas, planos, programas ou projetos na saúde de uma população, e na equidade em saúde (WHO, 1999). Com esta abordagem pretende-se identificar de que modo o processo de desenvolvimento produz efeitos nos fatores que condicionam a saúde e verificar as consequências nos resultados em saúde que daí possam advir (Quigley et al., 2006). As meIhores práticas de Avaliação de Impactos na Saúde são baseadas em princípios orientadores que assentam em valores de democracia, equidade, desenvolvimento sustentável, uso ético de evidência e aproximação global à saúde (WHO, 1999).

A Avaliação Ambiental Estratégica é um instrumento que, de forma estratégica, visa apoiar a criação de um contexto de desenvolvimento para a sustentabilidade, integrando as características do território e da sustentabilidade nos processos de avaliação de opções de desenvolvimento e nos processos de decisão (Partidário, 2012). Segundo Partidário (2012) esta abordagem de pensamento estratégico tem três objetivos: i) encorajar a integração ambiental e de sustentabilidade (incluindo os aspetos biofísicos, sociais, institucionais e económicos), estabelecendo as condições para acomodar futuras propostas de desenvolvimento, ii) acrescentar valor ao processo de decisão, discutindo as oportunidades e os riscos das opções de desenvolvimento e transformando problemas em oportunidades, iii) alterar mentalidades e criar uma cultura estratégica no processo de decisão, promovendo a cooperação e o diálogo institucionais e evitando conflitos. Tendo em conta a mesma autora (Partidário, 2012), apesar de este instrumento ter origem nos processos de avaliação de impacto, diferencia-se destes por procurar, através da avaliação, a capacidade de criar contextos de desenvolvimento que sejam favoráveis a soluções de sustentabilidade em vez de avaliar os efeitos do desenvolvimento no ambiente. 
No quadro 2 apresenta-se um processo de Avaliação Ambiental Estratégica semelhante aos processos de Avaliação de Impacto Ambiental, tal como defendido por vários autores, em seis etapas principais (Diretiva Europeia, 2001/42/CE) (quadro 2).

Quadro 2. Etapas da Avaliação Ambiental Estratégica semelhante à Avaliação de Impacto Ambiental

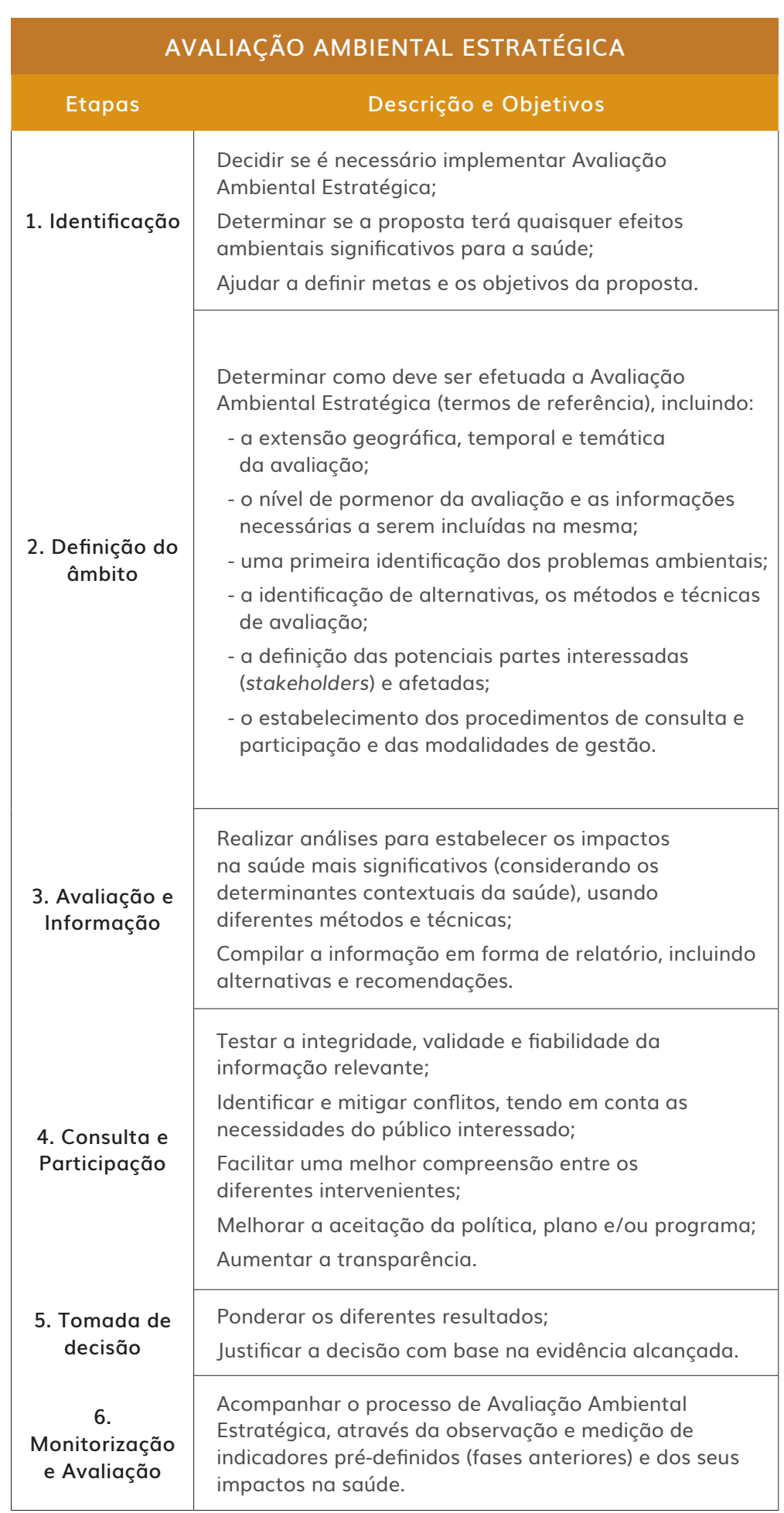

Segundo Partidário (2012) a Avaliação Ambiental Estratégica destina-se a avaliar as condições de desenvolvimento que permitem desencadear soluções de sustentabilidade. Deste modo a autora propõe apenas três fases fundamentais, tal como apresentado no quadro 3: i) compreender o contexto e focagem estratégica, ii) definir caminhos para a sustentabilidade e iii) promover a continuidade do processo de avaliação através de um programa de seguimento.

Com a Avaliação Ambiental Estratégica é possível identificar oportunidades para prevenir a doença e promover a saúde (Quigley et al., 2006; Fischer, Matuzzi \& Nowacki, 2010). O envolvimento de atores locais (stakeholders), e uma abordagem estratégica tal como promovida por Partidário (2012) garantem a integração de todos os interesses e interessados durante o processo de avaliação no longo prazo, considerando o modo como ações de desenvolvimento podem afetar a saúde humana. Assim, estas abordagens permitem avaliar os potenciais efeitos e impactos de dada proposta de política, plano e/ou programa sobre a saúde, partindo dos determinantes contextuais da saúde (Quigley et al., 2006).

No início do século $X X$, estes instrumentos de avaliação de impactos - Avaliação de Impactos na Saúde e Avaliação Ambiental Estratégica - surgem como uma oportunidade para a aplicação da ação intersectorial na saúde. Esta ação baseia-se na evidência de que ações e política fora do exclusivo domínio do sector da saúde têm repercussões na saúde e na equidade em saúde (Partidário \& Jesus, 2007; Nowacki, Martuzzi \& Fischer, 2009; Santana, 2014). 


\begin{tabular}{|c|c|}
\hline \multicolumn{2}{|c|}{ AVALIAÇÃO AMBIENTAL ESTRATÉGICA } \\
\hline Etapas & Descrição e Objetivos \\
\hline \multirow{3}{*}{$\begin{array}{l}\text { 1) Contexto } \\
\text { da Avaliação } \\
\text { Ambiental } \\
\text { Estratégica e } \\
\text { foco estratégico }\end{array}$} & Estabelece o contexto e a focagem estratégica. \\
\hline & $\begin{array}{l}\text { A finalidade é assegurar que a avaliação se concentra } \\
\text { apenas no que é importante, que compreende e se } \\
\text { adapta ao contexto natural, social, cultural, político e } \\
\text { económico do objeto em avaliação. }\end{array}$ \\
\hline & $\begin{array}{l}\text { Tem como objetivo criar um marco de avaliação } \\
\text { estratégico com base em Fatores Críticos de Decisão. }\end{array}$ \\
\hline $\begin{array}{l}\text { 2) Caminhos e } \\
\text { diretrizes para a } \\
\text { sustentabilidade }\end{array}$ & $\begin{array}{l}\text { Refere-se à criação de caminhos para a sustentabilidade, } \\
\text { e diretrizes que apoiem esse percurso. Caminhos para } \\
\text { a sustentabilidade é o termo usado para exprimir as } \\
\text { opções estratégicas para o desenvolvimento, que nos } \\
\text { ajudam a ir de onde estamos até onde queremos chegar }\end{array}$ \\
\hline $\begin{array}{l}\text { 3) Uma fase } \\
\text { contínua de } \\
\text { seguimento, } \\
\text { ligação de } \\
\text { processos e } \\
\text { envolvimento }\end{array}$ & $\begin{array}{l}\text { O seguimento, com monitorização, avaliação e } \\
\text { comunicação, deve ser um ato contínuo, ou de } \\
\text { rotina, num processo de avaliação ambiental e de } \\
\text { sustentabilidade estratégica, sistematicamente ligado } \\
\text { aos processos de formulação de políticas ou de } \\
\text { planeamento e envolvendo os agentes relevantes. }\end{array}$ \\
\hline
\end{tabular}

Fonte: Partidário, 2012:33-34 ções da estratégia, intervenção e decisão política para a saúde mental (Todman et al., 2012).

Todos os fatores elencados se encontram intimamente relacionados com os determinantes contextuais da saúde mental, podendo ser influenciados por eles, assim como com os conceitos de equidade e justiça social (Lalani, 2011). Neste sentido, os processos de avaliação estratégica de impactos na saúde mental deverão ter em conta a análise do indivíduo, da comunidade e do território, potenciando uma compreensão estratégica e integrada dos fenómenos, que permitirá apoiar os processos de tomada de decisão a nível local.
Em Portugal, a Direção-Geral da Saúde (2009) sensível a esta temática, preparou uma circular informativa com recomendações para a integração e apreciação da "saúde" ao nível dos instrumentos de gestão territorial municipais, nomeadamente Planos Municipais de Ordenamento do Território. O seu objetivo é "contribuir para um correto ordenamento do território municipal, na perspetiva de que a qualidade de vida das populações passa em primeira instância pela sua saúde e bem-estar" (Circular informativa $n^{\circ} 36 /$ DA de 9 de outubro de 2009, p.2).

Tendo como objetivo a promoção e proteção da saúde mental, a implementação de um instrumento de avaliação estratégica de impactos na saúde mental deverá ainda considerar quatro fatores orientadores: i) aumentar/reforçar o controlo por parte da comunidade (oportunidades para fazer escolhas saudáveis), ii) aumentar a capacidade de resiliência e as valências da comunidade, iii) facilitar a participação e iv) promover a inclusão (Cooke et al., 2011; Lalani, 2011). Não existindo saúde sem saúde mental, e sendo a saúde mental parte integrante da saúde geral de um indivíduo ou comunidade (WHO, 2010), estas abordagens de avaliação de impac-

\section{Considerações finais}

As características do contexto (onde os indivíduos nascem, crescem, trabalham e passam os tempos livres) influenciam os resultados em saúde mental das populações. A literatura científica sustenta que os ambientes físico, construído, socioeconómico e de interação social e cultural produzem impactos no bem-estar individual e da comunidade. A ocorrência de determinadas doenças mentais é assim justificada como consequência das características dos lugares, sendo esta relação ainda mais expressiva e significativa em períodos de crise económica.

A identificação das características contextuais, que podem influenciar a vida humana, e a sua medição e monitorização é um dos grandes desafios colocados a quem intervêm no território, no desenho de intervenções de planeamento saudável (Santana, 2009). A adoção de abordagens de avaliação estratégica de impactos pode contribuir, de forma integrada e participada, para um melhor entendimento dos fatores individuais, ambientais, sociais e institucionais que 
influenciam a saúde mental e das suas desigualdades (Macintyre \& Ellaway, 2000; Fone \& Dunstan, 2006; Sundquist \& Ahlen, 2006; Partidário \& Jesus, 2007; Mair, Diez Roux \& Galea, 2008; Paczkowski \& Galea, 2010).

Em contextos de crise económica, a importância atribuída a estes processos e mecanismos de avaliação de impactos ainda é maior, uma vez que permitem perspetivas e análises objetivas sobre o aumento das situações de vulnerabilidade e de desigualdade, apesar da escassez de recursos. Os impactos da perda de saúde mental em períodos de crise são enormes para os indivíduos afetados, para os empregadores e para a sociedade em geral. Num relatório publicado recentemente pela OCDE (2015) o custo da saúde mental estimado é, em média, 3,5\% do PIB dos seus estados membros (33 países), tendo em conta a perda potencial de força laboral, o aumento de taxas de desemprego e de laboral, bem como a perda de produtividade laboral.

Uma série de recomendações são apresentadas no mesmo relatório ao nível da política social e de saúde, reconhecendo-se que a perda de recursos económicos e de produtividade está associada a indivíduos com problemas de saúde de gravidade baixa ou média. Como tal, intervenções atempadas são referidas como o método mais eficaz para prevenir o desenvolvimento de problemas de saúde mental. Exemplos de políticas públicas eficazes neste domínio seriam, por exemplo, o aumento das competências de profissionais fora da área da saúde em questões de saúde mental, através da elaboração e distribuição de guias de melhores práticas para identificar e referenciar indivíduos com sinais de eventuais problemas mentais, considerando também a evolução sintomatológica. É sugerido também a identificação de responsabilidades profissionais dos que lidam com casos de saúde mental, tendo em conta a sua atuação no tratamento de indivíduos. Finalmente, a implementação das boas práticas deverá ser suportada por um sistema de monitorização, que deverá incluir mecanismos sancionatórios para profissionais que não atuem em conformidade com o estabelecido (OECD, 2015). A associação entre choques económicos e degra- dação da saúde mental não é inevitável e pode ser quebrada ao atuar sobre os mecanismos sociais que medeiam essa associação, com destaque para intervenções políticas de proteção social e laboral e políticas de prevenção ativa e de proteção do acesso a cuidados de saúde atempados e de qualidade.

Em tempos de crise deve ser reforçada a criação de evidência científica sobre os fatores condicionantes da saúde mental, com base numa abordagem compreensiva, transversal e estratégica da saúde, de modo a apoiar os decisores-políticos e a suportar as intervenções no território. Neste âmbito, a observação e análise do Lugar, enquanto espaço apropriado, vivido e sentido pelo homem, é fundamental para assegurar e promover a saúde e o bem-estar dos indivíduos e das suas comunidades (Cresswell, 2004; Cummins et al., 2007), porque todos têm direito a viver, trabaIhar e ocupar o seu tempo livre em locais e comunidades que sejam seguras, saudáveis e livres de quaisquer ameaças (Corburn, 2004).

\section{Agradecimentos}

Este estudo foi desenvolvido no âmbito do projeto de investigação PTDC/ATP-GEO/4101/2012, SMAILE, Saúde Mental - Avaliação do Impacto das Condicionantes Locais e Económicas, e da bolsa de doutoramento SFRH/ BD/92369/2013, financiados por Fundos FEDER através do Programa Operacional Factores de Competitividade COMPETE e por Fundos Nacionais através da FCT - Fundação para a Ciência e a Tecnologia.

Agradece-se ao grupo de investigação do projeto SMAILE (Benedetto Saraceno, Carla Nunes, Graça Cardoso, João Ferrão, José Caldas de Almeida, Manuela Silva, Pedro Pita Barros) pelos contributos ao longo destes dois anos de desenvolvimento do projeto. 


\section{Referências Bibliográficas}

Almedom, A. M. (2005). Social capital and mental health: an interdisciplinary review of primary evidence. Social Science and Medicine, 61, 943-964.

Annerstedt, M., Ostergren, P.-O., Björk, J., Grahn, P., Skärbäck, E., \& Währborg, P. (2012). Green qualities in the neighbourhood and mental health - results from a longitudinal cohort study in Southern Sweden. BMC Public Health, 12, 337. doi:10.1186/1471-2458-12-337

Araya, R., Dunstan, F., Playle, R., Thomas, H., Palmer, S., \& Lewis, G. (2006). Perceptions of social capital and the built environment and mental health. Social Science \& Medicine, 62, 3072-83. doi:10.1016/j.socscimed.2005.11.037

Araya, R., Montgomery, A., Rojas, G., Fritsch, R., Solis, J., Signorelli, A., \& Lewis, G. (2007). Common mental disorders and the built environment in Santiago, Chile. The British Journal of Psychiatry: The Journal of Mental Science, 190, 394-401. doi:10.1192/bjp.bp.106.024596

Artazcoz, L., Benach, J., Borrell, C., \& Cortès, I. (2004). Unemployment and mental health: understanding the interactions among gender, family roles, and social class. American Journal of Public Health, 94(1), 82-88. doi:10.2105/AJPH.94.1.82

Barahmand, U., Shahbazi, H., \& Shahbazi, Z. (2013). Implications of perceived physical and social aspects of the environment for self-reported physical and mental health. International Journal of Environmental Health Research, 23(1), 31-45.

Barcellos, C., \& Bastos, F. I. (1996). Geoprocessamento, ambiente e saúde: uma união possível? Cadernos de Saúde Pública, 12(3), 389-397. doi:10.1590/S0102$311 \times 1996000300012$

Barlow, P., Reeves, A., McKeee, M., \& Stuckler, D. (2015). Austerity, precariousness, and the health status of Greek labour market participants: Retrospective cohort analysis of employed and unemployed persons in 2008-2009 and 2010-2011. Journal of Public Health Policy, 36(4), 452-68.

Barton, H., Grant, M., \& Guise, R. (2003). Shaping Neighbourhoods - a guide for health, sustainability and vitality (Spon Press). New York.

Baum, F. E., \& Ziersch, A. M. (2003). Social capital. Journal of Epidemiology and Community Health, 57, 320323. doi:10.1136/jech.57.5.320

Berke, E. M., Gottlieb, L. M., Moudon, A. V., \& Larson, E. B. (2007). Protective association between neighborhood walkability and depression in older men. Journal of the American Geriatrics Society, 55(4), 52633. doi:10.1111/j.1532-5415.2007.01108.x

Birley, M. (2011). Health Impact Assessment. Principles and Practice (Earthscan, p. 356). Nova lorque.
Bond, L., Kearns, A., Mason, P., Tannahill, C., Egan, M., \& Whitely, E. (2012). Exploring the relationships between housing, neighbourhoods and mental wellbeing for residents of deprived areas. BMC Public Health, 12(48), 1-14.

Bor, J., Basu, S., Coutts, A., Mckee, M., \& Stuckler, D. (2013). Alcohol use during the great recession of 2008-2009. Alcohol and Alcoholism, 48(3), 343-348. doi:10.1093/alcalc/agt002

Breslin, F. C., \& Mustard, C. (2003). Factors influencing the impact of unemployment on mental health among young and older adults in a longitudinal, population-based survey. Scandinavian Journal of Work Environment \& Health, 29, 5-14.

Brown, J., Learmonth, A., \& Mackereth, C. (2015). Promoting Public Mental Health and Well-being: Principles into Practice (p. 304). London: Jessica Kingsley Publishers.

Brown, P. (1995). Race, class, and environmental health: a review and systematization of the literature. Environmental Research, 69, 15-30. doi:10.1006/enrs.1995.1021

Brown, S. C., Mason, C. A., Lombard, J. L., Martinez, F., Plater-Zyberk, E., Spokane, A. R., Szapocznik, J. (2009). The relationship of built environment to perceived social support and psychological distress in Hispanic elders: the role of "eyes on the street". The Journals of Gerontology. Series B, Psychological Sciences and Social Sciences, 64(2), 234-46. doi:10.1093/geronb/gbn011

Burton, L. (2015). Mental Well-being and the Influence of Place. In H. Barton, S. Thompson, S. Burgess, \& M. Grant (Eds.), The Routledge Handbook of Planning for Health and Well-Being: Shaping a sustainable and healthy future (Routledge, p. 617). Oxon: Taylor \& Francis.

Cannuscio, C. C., Weiss, E. E., \& Asch, D. A. (2010). The Contribution of Urban Foodways to Health Disparities. Journal of Urban Health, 87(3), 381-393. doi:10.1007/ s11524-010-9441-9

Catalano, R., \& Bellows, B. (2005). Commentary: If economic expansion threatens public health, should epidemiologists recommend recession? International Journal of Epidemiology, 34(6), 1212-1213. doi:10.1093/ije/dyi145

Chou, K. L. (2012). Perceived discrimination and depression among new migrants to Hong Kong: The moderating role of social support and neighborhood collective efficacy. Journal of Affective Disorders, 138(1), 63-70. doi:10.1016/j.jad.2011.12.029

Comino, E. J., Harris, E., Chey, T., Manicavasagar, V., Penrose Wall, J., Powell Davies, G., \& Harris, M. F. (2003). Relationship between mental health disorders and unemployment status in Australian adults. The Australian and New Zealand Journal of Psychiatry, 37(2), 230-235. doi:10.1046/j.1440-1614.2003.01127.x 
Cooke, A., Friedli, L., Coggins, T., Edmonds, N., Michaelson, J., O'Hara, K., Scott-Samuel, A. (2011). Mental Well-being Impact Assessment: A toolkit for well-being (p. 141). London.

Corburn, J. (2004). Confronting the challenges in reconnecting urban planning and public health. American Journal of Public Health, 94(4), 541-546. doi:10.2105/ AJPH.94.4.541

Córdoba-Doña, J., San Sebastián, M., Escolar-Pujolar, A., Martínez-Faure, J. E., \& Gustafsson, P. (2014). Economic crisis and suicidal behaviour: the role of unemployment, sex and age in Andalusia, southern Spain. International Journal for Equity in Health, 13(1), 55. doi:10.1186/1475-9276-13-55

Corrêa, A., Moreira-Almeida, A., Menezes, P., Vallada, H., \& Scazufca, M. (2010). Investigating the role played by social support in the association between religiosity and mental health in low income older adults: results from the São Paulo Ageing \& Health Study (SPAH). Revista Brasileira de Psiquiatria, 33(2), 157-164.

Cresswell, T. (2004). Place: A Short Introduction (p. 168). Wiley-Blackwell. doi:10.1139/h2012-055

Cromley, E., Wilson-Genderson, M., \& Pruchno, R. (2012). Neighborhood characteristics and depressive symptoms of older people: Local spatial analyses. Social Science and Medicine, 75(12), 2307-2316. doi:10.1016/j.socscimed.2012.08.033

Cummins, S., Curtis, S., Diez-Roux, A. V., \& Macintyre, S. (2007). Understanding and representing "place" in health research: A relational approach. Social Science and Medicine, 65(2007), 1825-1838. doi:10.1016/j.socscimed.2007.05.036

Curtis, S. (2010). Space, place and mental health. Geographies of health. Surrey: Ashgate.

De Snyder, V., Friel, S., Fotso, J. C., Khadr, Z., Meresman, S., Monge, P., \& Patil-Deshmukh, A. (2011). Social conditions and urban health inequities: realities, challenges and opportunities to transform the urban landscape through research and action. Journal of Urban Health Bulletin of the New York Academy of Medicine, 88(6), 1183-93. doi:10.1007/s11524-011-9609-y

Drukker, M., \& van Os, J. (2003). Mediators of neighbourhood socioeconomic deprivation and quality of life. Social Psychiatry Psychiatr Epidemiol, 38(12), 698-706.

Dupéré, V., \& Perkins, D. D. (2007). Community types and mental health: A multilevel study of local environmental stress and coping. American Journal of Community Psychology, 39(1), 107-119. doi:10.1007/s10464-007-9099-y

Evans, G. W. (2003). The built environment and mental health. Journal of Urban Health: Bulletin of the New York Academy of Medicine, 80(4), 536-55. doi:10.1093/ jurban/jtg063
Evans, G, Wells, N., \& Moch, A. (2003). Housing and mental health: a review of the evidence and a methodological and conceptual critique. Journal of Social Issues, 59(3), 475-500. doi:10.1111/1540-4560.00074

Fischer, T., Matuzzi, M., \& Nowacki, J. (2010). The consideration of health in strategic environmental assessment (SEA). Environmental Impact Assessment Review, 30, 200-210. doi:10.1016/j.eiar.2009.10.005

Fone, D., Dunstan, F., John, A., \& Lloyd, K. (2007). Associations between common mental disorders and the Mental Illness Needs Index in community settings. Multilevel analysis. The British Journal of Psychiatry: The Journal of Mental Science, 191(2007), 158-163. doi:10.1192/bjp.bp.106.027458

Fone, D., Dunstan, F., Williams, G., Lloyd, K., \& Palmer, S. (2007). Places, people and mental health: a multilevel analysis of economic inactivity. SocialScience \& Medicine, 64(3), 633-45. doi:10.1016/j.socscimed.2006.09.020

Fone, D. L., \& Dunstan, F. D. J. (2006). Mental health, places and people: a multilevel analysis of economic inactivity and social deprivation. Health \& Place, 12(3), 332-344.

Fukuda, Y., \& Hiyoshi, A. (2012). Influences of income and employment on psychological distress and depression treatment in Japanese adults. Environmental Health and Preventive Medicine, 17(1), 10-17. doi:10.1007/s12199-011-0212-3

Galea, S., Ahern, J., Rudenstine, S., Wallace, Z., \& Vlahov, D. (2005). Urban built environment and depression: a multilevel analysis. Journal of Epidemiology and Community Health, 59(10), 822-7. doi:10.1136/ jech.2005.033084

Gary, T. L., Stark, S. A., \& LaVeist, T. A. (2007). Neighborhood characteristics and mental health among African Americans and whites living in a racially integrated urban community. Health \& Place, 13, 569575. doi:10.1016/j.healthplace.2006.06.001

Gili, M., Roca, M., Basu, S., McKee, M., \& Stuckler, D. (2012). The mental health risks of economic crisis in Spain: evidence from primary care centres, 2006 and 2010. The European Journal of Public Health, 1-5. doi:10.1093/eurpub/cks035

Guite, H. F., Clark, C., \& Ackrill, G. (2006). The impact of the physical and urban environment on mental well-being. Public Health, 120(12), 1117-26. doi:10.1016/j. puhe.2006.10.005

Hamano, T., Fujisawa, Y., Ishida, Y., Subramanian, S. V., Kawachi, I., \& Shiwaku, K. (2010). Social capital and mental health in Japan: A multilevel analysis. PLOS ONE, 5 (10). doi:10.1371/journal.pone.0013214 
Harhay, M., Bor, J., Basu, S., Mckee, M., Mindell, J., Shelton, N., \& Stuckler, D. (2014). Differential impact of the economic recession on alcohol use among white British adults, 2004-2010. European Journal of Public Health, 24(3), 410-415. doi:10.1093/eurpub/ckt134

Harris-roxas, B., Viliani, F., Bond, A., Cave, B., Divall, M., Furo, P., Winkler, M. (2012). Impact Assessment and Project Appraisal Health impact assessment: the state of the art. Impact Assessment and Project Appraisal, 30(1), 45-55.

Harris-Roxas, B., Viliani, F., Bond, A., Cave, B., Divall, M., Furu, P., Winkler, M. (2012). Health impact assessment: the state of the art. Impact Assessment and Project Appraisal, 30(1). doi:10.1080/14615517.2012.666035

Hempstead, K. (2006). The geography of self-injury: spatial patterns in attempted and completed suicide. Social Science \& Medicine (1982), 62(12), 3186-96. doi:10.1016/j.socscimed.2005.11.038

Huurre, T., Rahkonen, O., Komulainen, E., \& Aro, H. (2005). Socioeconomic status as a cause and consequence of psychosomatic symptoms from adolescence to adulthood. Social Psychiatry and Psychiatric Epidemiology, 40(7), 580-587. doi:10.1007/s00127-005-0930-1

Jagodic, H., Agius, M., \& Pregelj, P. (2012). Inter-regional variations in suicide rates. Psychiatria Danubina, 24(1), 82-85.

Kaplan, G. (1996). People and places: contrasting perspectives on the association between social class and health. International Journal of Health Services: Planning, Administration, Evaluation, 26(3), 507-519. doi:10.2190/4CUU-7B3G-G4XR-0K0B

Kubzansky, L., Subramanian, S., Kawachi, I., Fay, M., Soobader, M., \& Berkman, L. (2005). Neighborhood contextual influences on depressive symptoms in the elderly. American Journal of Epidemiology, 162(3), 253260. doi:10.1093/aje/kwi185

Lalani, N. (2011). Mental Well-being Impact Assessment: A Primer (p. 8).

Lee, M. (2009). Neighborhood residential segregation and mental health: A multilevel analysis on Hispanic Americans in Chicago. Social Science and Medicine, 68(11), 1975-1984. doi:10.1016/j.socscimed.2009.02.040

Lorant, V., Croux, C., Weich, S., Deliège, D., Mackenbach, J., \& Ansseau, M. (2007). Depression and socio-economic risk factors: 7-year longitudinal population study. The British Journal of Psychiatry: The Journal of Mental Science, 190(4), 293-298. doi:10.1192/bjp.bp.105.020040

Ludwig, J., Duncan, G., Gennetian, L., Katz, L., Kessler, R., Kling, J., \& Sanbonmatsu, L. (2012). Neighborhood Effects on the Long-Term Well-Being of Low-Income Adults. Science, 337 (6101), 1505-1510. doi:10.1126/science. 1224648
Macintyre, S., \& Ellaway, A. (2000). Ecological Approaches: Rediscovering the Role of the Physical and Social Environment. Social Epidemiology, 9(5), 332-348. Oxford University Press.

Mair, C., Diez Roux, A. V, \& Galea, S. (2008). Are neighbourhood characteristics associated with depressive symptoms? A review of evidence. Journal of Epidemiology \& Community Health, 62, 940-946.

Miles, R., Coutts, C., \& Mohamadi, A. (2012). Neighborhood urban form, social environment, and depression. Journal of Urban Health, 89(1), 1-18. doi:10.1007/s11524-011-9621-2

Murali, V. (2004). Poverty, social inequality and mental health. Advances in Psychiatric Treatment, 10(3), 216224. doi:10.1192/apt.10.3.216

Murata, C., Kondo, K., Hirai, H., Ichida, Y., \& Ojima, T. (2008). Association between depression and socio-economic status among community-dwelling elderly in Japan: The Aichi Gerontological Evaluation Study (AGES). Health \& Place, 14(3), 406-414. doi:10.1016/j.healthplace.2007.08.007

Murphy, G., \& Athanasou, J. (1999). The effect of unemployment on mental health. Journal of Occupational and Organizational Psychology, 72(1), 83-99. doi:10.1348/096317999166518

Myer, L., Stein, D., Grimsrud, A., Seedat, S., \& Williams, D. (2008). Social determinants of psychological distress in a nationally-representative sample of South African adults. Social Science and Medicine, 66(8), 1828-1840. doi:10.1016/j.socscimed.2008.01.025

Nogueira, H. (2006). Os Lugares e a Saúde. Universidade de Coimbra.

Nogueira, H. (2007). Territórios de Privação Múltipla na Área Metropolitana de Lisboa. In P. Santana (Ed.), A Cidade e a Saúde (pp. 133-141). Coimbra: Edições Almedina.

Nogueira, H., Santana, P., \& Santos, R. (2007). Saúde: Vulnerabilidade e Oportunidade na Àrea Metropolitana de Lisboa. In P. Santana (Ed.), A Cidade e a Saúde (pp. 119-132). Coimbra: Edições Almedina.

Nowacki, J., Matuzzi, M., \& Fischer, T. (2009). Health and strategic environmental assessment (p. 90). Copenhagen, Denmark.

OECD. (2015). Fit Mind, Fit Job. From evidence to practice in mental health and work (p. 175). Paris.

Orpana, H., Lemyre, L., \& Gravel, R. (2009). Income and psychological distress: The role of the social environment. Statistics Canada Health Reports, 20(1), 1-8.

Ostir, G., Eschbach, K., Markides, K., \& Goodwin, J. (2003). Neighbourhood composition and depressive symptoms among older Mexican Americans. Journal of 
Epidemiology and Community Health, 57(12), 987-992. doi:10.1136/jech.57.12.987

Paczkowski, M., \& Galea, S. (2010). Sociodemographic characteristics of the neighborhood and depressive symptoms. Current Opinion in Psychiatry, 23(4), 337-341.

Partidário, M. do R. (2012). Guia de melhores práticas para Avaliação Ambiental Estratégica - orientações metodológicas para um pensamento estratégico em AAE (p. 75). Lisboa.

Partidário, M. do R., \& Jesus, J. (2003). Fundamentos da Avaliação do Impacte Ambiental (p. 252). Lisboa: Universidade Aberta.

Partidário, M. do R., \& Jesus, J. (2007). A Avaliação de Impactes na Saúde. In P. Santana (Ed.), A Cidade e a Saúde (pp. 55-68). Coimbra: Edições Almedina.

Patel, V., Lund, C., Hatherill, S., Plagerson, S., Corrigall, J., Funk, M., \& Flisher, A. J. (2010). Mental disorders: equity and social determinants. In A. Blas \& A. S. Kurup (Eds.), Equity, Social Determinants and Public Health Programs (pp. 115-134). Genebra: World Health Organization.

Peen, J., Dekker, J., Schoevers, R., Have, M., de Graaf, R., \& Beekman, A. (2007). Is the prevalence of psychiatric disorders associated with urbanization? Social Psychiatry and Psychiatric Epidemiology, 42(12), 984-989. doi:10.1007/s00127-007-0256-2

Poblete, F. C., Sapag, J. C., \& Bossert, T. J. (2008). Capital social y salud mental en comunidades urbanas de nivel socioeconómico bajo, em Santiago, Chile. Nuevas formas de entender la relacióon comunidad-salud. Revista Médica de Chile, 136(2), 230-9. doi:/S003498872008000200014

Prince, M., Patel, V., Saxena, S., Maj, M., Maselko, J., Phillips, M. R., \& Rahman, A. (2007). No health without mental health. The Lancet, 370(9590), 859-877. doi:10.1016/S0140-6736(07)61238-0

Pringle, D., Cook, S., Poole, M., \& Moore, A. (2000, January 1). Cross-Border Deprivation Analysis: a Summary Guide. Oak Tree Press. Retrieved from http://eprints. ulster.ac.uk/31917/

Quigley, R., Broeder, L. den, Furu, P., Bond, A., Cave, B., \& Bos, R. (2006). Health Impact Assessment. International Best Practice Principles. Fargo, USA: International Association for Impact Assessment, 5, 5-8.

Quinn, N., \& Biggs, H. (2010). Creating partnerships to improve community mental health and well-being in an area of high deprivation: lessons from a study with highrise flat residents in east Glasgow. Journal of Public Mental Health, 9(4), 16-21. doi:10.5042/ jpmh.2010.0699
Rios, R., Aiken, L., \& Zautra, A.(2012). Neighborhood contexts and the mediating role of neighborhood social cohesion on health and psychological distress among hispanic and non-hispanic residents. Annals of Behavioral Medicine, 43(1), 50-61. doi:10.1007/s12160-011-9306-9

Roberts, B., Abbott, P., \& Mckee, M. (2010). Levels and determinants of psychological distress in eight countries of the former Soviet Union. Journal of Public Mental Health, 9(3), 17-26.

Ruhm, C. (2000). Are recessions good for your health? The Quarterly Journal of Economics, 115(2), 617-650.

Ruhm, C. (2003). Good times make you sick. Journal of Health Economics, 22(4), 637-58. doi:10.1016/S01676296(03)00041-9

Ruhm, C. (2005). Healthy living in hard times. Journal of Health Economics, 24(2005), 341-363.

Santana, P. (2005). Geografias da Saúde e do Desenvolvimento - Evolução e Tendências em Portugal (p. 342). Coimbra: Almedina.

Santana, P. (2009). Por uma Cidade Saudável. Janus, 1-7. Santana, P. (2014). Introdução à Geografia da Saúde. Território, Saúde e Bem-estar (p. 192). Coimbra: Imprensa da Universidade de Coimbra.

Santana, P., Costa, C., Cardoso, G., Loureiro, A., \& Ferrão, J. (2015). Suicide in Portugal: Spatial determinants in a context of economic crisis. Health \& Place, 35, 8594. doi:10.1016/j.healthplace.2015.07.001

Santana, P., Costa, C., \& Loureiro, A. (2014). Os sistemas de informação geográfica e o planeamento urbano saudável na Amadora. Revista Do Departamento de Geografia - USP, Volume Esp, 368-389.

Santana, P., Santos, R., \& Nogueira, H. (2009). The link between local environment and obesity: a multilevel analysis in the Lisbon Metropolitan Area, Portugal. Social Science \& Medicine, 68(4), 601-609. doi:10.1016/j. socscimed.2008.11.033

Semenza, J., \& Krishnasamy, P. (2007). Design of a health-promoting neighborhood intervention. Health Promotion Practice, 8(3), 243-56. doi:10.1177/1524839906289585

Sheppard, A., Salmon, C., Balasubramaniam, P., Parsons, J., Singh, G., Jabbar, A., O'Campo, P. (2012). Are residents of downtown Toronto influenced by their urban neighbourhoods? Using concept mapping to examine neighbourhood characteristics and their perceived impact on self-rated mental well-being. International Journal of Health Geographics, 11(31). doi:10.1186/ 1476-072X-11-31 
Skapinakis, P., Lewis, G., Araya, R., Jones, K., \& Williams, G. (2005). Mental health inequalities in Wales, UK: multi-level investigation of the effect of area deprivation. The British Journal of Psychiatry: The Journal of Mental Science, 186(5), 417-22. doi:10.1192/bjp.186.5.417

Stafford, M. (2003). Neighbourhood deprivation and health: does it affect us all equally? International Journal of Epidemiology, 32(3), 357-366. doi:10.1093/ije/dyg084

Stuckler, D., \& Basu, S. (2013). The Body Economic: Why Austerity Kills. New York: Basic Books.

Stuckler, D., Basu, S., Suhrcke, M., Coutts, A., \& McKee, M. (2009). The public health effect of economic crises and alternative policy responses in Europe: an empirical analysis. The Lancet, 374(9686), 315-323. doi:10.1016/ s0140-6736(09)61124-7

Sugiyama, T., Leslie, E., Giles-Corti, B., \& Owen, N. (2008). Associations of neighbourhood greenness with physical and mental health: do walking, social coherence and local social interaction explain the relationships? Journal of Epidemiology and Community Health, 62, e9. doi:10.1136/jech.2007.064287

Sundquist, K., \& Ahlen, H. (2006). Neighbourhood income and mental health: a multilevel follow-up study of psychiatric hospital admissions among 4.5 million women and men. Health \& Place, 12(4), 594-602.

Takano, T. (2002). Urban residential environments and senior citizens' longevity in megacity areas: the importance of walkable green spaces. Journal of Epidemiology \& Community Health, 56(12), 913-918. doi:10.1136/ jech.56.12.913

Thomas, C., Benzeval, M., \& Stansfeld, S. (2007). Psychological distress after employment transitions: the role of subjective financial position as a mediator. Journal of Epidemiology and Community Health, 61(1), 48-52. doi:10.1136/jech.2005.044206

Thomas, H., Weaver, N., Patterson, J., Jones, P., Bell, T., Playle, R., Araya, R. (2007). Mental health and quality of residential environment. The British Journal of Psychiatry: The Journal of Mental Science, 191(6), 500-5. doi:10.1192/bjp.bp.107.039438

Todman, L., Hricisak, L., Fay, J., \& Sherrod Taylor, J. (2012). Mental health impact assessment: population mental health in Englewood, Chicago, Illinois, USA. Impact Assessment and Project Appraisal, 30(2), 116-123. doi:10.1080/14615517.2012.659991

Todman, L., Taylor, J. S., McDowell, T., Driscoll, M., Cooper, D., \& Kim, E. (2013). What are the Social Determinants of Mental Health? Retrieved October 6, 2015, from http:// www.adler.edu/page/institutes/institute-on-social-exclusion/projects/mhia/social-determinants-of-mental-health
Tsai, J., \& Thompson, E. (2013). Impact of Social Discrimination, Job Concerns, and Social Support on Filipino Immigrant Worker Mental Health and Substance Use. American Journal of Industrial Medicine, 56(9), 1082-1094.

Van Ryn, M., \& Vinokur, a D. (1992). How did it work? An examination of the mechanisms through which an intervention for the unemployed promoted job-search behavior. American Journal of Community Psychology, 20(5), 577-97.

Vinokur, A., Schul, Y., Vuori, J., \& Price, R. (2000). Two years after a job loss: long-term impact of the JOBS program on reemployment and mental health. Journal of occupational health psychology, 7(4), 302-312. doi:10.1037/1076-8998.5.1.32

Vlahov, D., Galea, S., Gibble, E., \& Freudenberg, N. (2005). Perspectives on urban conditions and population health Perspectivas sobre condições urbanas e saúde da população. Cadernos de Saúde Pública, 21(3), 949-957.

Vuori, J., Silvonen, J., Vinokur, A. D., \& Price, R. H. (2002). The Työhön Job Search Program in Finland: Benefits for the unemployed with risk of depression or discouragement. Journal of Occupational Health Psychology, 7(1), 5-19. doi:10.1037//1076-8998.7.1.5

Walters, K., Breeze, E., Wilkinson, P., Price, G. M., Bulpitt, C. J., \& Fletcher, A. (2004). Local area deprivation and urban-rural differences in anxiety and depression among people older than 75 years in Britain. American Journal of Public Health, 94(10), 1768-1774. doi:10.2105/AJPH.94.10.1768

Weich, S., Blanchard, M., Prince, M., Burton, E., Erens, B., \& Sproston, K. (2002). Mental health and the built environment: cross-sectional survey of individual and contextual risk factors for depression. The British Journal of Psychiatry: The Journal of Mental Science, 180(5), 428-433. doi:10.1192/bjp.180.5.428

Whitley, R., \& Prince, M. (2005). Fear of crime, mobility and mental health in inner-city London, UK. Social Science and Medicine, 61(8), 1678-1688. doi:10.1016/j. socscimed.2005.03.044

WHO. (1999). Health impact assessment: main concepts and suggested approach (Gothenburg Consensus Paper). Brussels.

WHO. (2001). The World health report: 2001: Mental health: new understanding, new hope (p. 178). Geneva.

WHO. (2008). Closing the gap in a generation. health equity through action on the social determinants of health (p. 246). doi:10.1080/17441692.2010.514617

WHO. (2010). Mental health: strengthening our response. Fact sheet 220. 
WHO. (2013). Review of social determinants and the health divide in the WHO European Region: final report (p. 188). Denmark (Copenhagen).

WHO. (2014). Mental health: a state of well-being. Retrieved October 6, 2015, from http://www.who.int/features/factfiles/mental_health/en/

WHO, \& Calouste Gulbenkian Foundation. (2014). Social Determinants of Mental Health (p. 52). Geneva.

Wilson-Genderson, M., \& Pruchno, R. (2013). Effects of neighborhood violence and perceptions of neighborhood safety on depressive symptoms of older adults. Social Science and Medicine, 85, 43-49. doi:10.1016/j.socscimed.2013.02.028

Yang, T.-C., \& Matthews, S. A. (2010). The role of social and built environments in predicting self-rated stress: A multilevel analysis in Philadelphia. Health \& Place, 16(5), 803-810. doi:10.1016/j.healthplace.2010.04.005

Zhang, J. X., Ho, S. C., \& Woo, J. (2005). Assessing mental health and its association with income and resource utilization in old-old Chinese in Hong Kong. The American Journal of Geriatric Psychiatry: Official Journal of the American Association for Geriatric Psychiatry, 13(3), 236-243. doi:10.1176/appi.ajgp.13.3.236

\section{Legislação e circulares informativas}

Diretiva 2001/42/CE: http://eur-lex.europa.eu/LexUriServ/LexUriServ.do?uri=OJ:L:2001:197:0030:0037:PT: PDF (consultada em 15 de outubro de 2015)

Circular informativa $n^{\circ}$ 36/DA de 9 de Outubro de 2009: http://www.apai.org.pt/m1/1255520754circulardgs36da091009.pdf (consultada em 15 de outubro de 2015) 